Georgian Mathematical Journal

Volume 13 (2006), Number 2, 215-228

\title{
EXISTENCE THEORY FOR PERTURBED NONLINEAR BOUNDARY VALUE PROBLEMS WITH INTEGRAL BOUNDARY CONDITIONS
}

\author{
ABDELKADER BELARBI, MOUFFAK BENCHOHRA, AND BAPURAO C. DHAGE
}

\begin{abstract}
In this paper, the existence of solutions and extremal solutions for a second order perturbed nonlinear boundary value problem with integral boundary conditions is proved under the mixed generalized Lipschitz and Carathéodory conditions.
\end{abstract}

2000 Mathematics Subject Classification: 34A60, 34B15.

Key words and phrases: Nonlinear boundary value problem, fixed point, extremal solutions.

\section{INTRODUCTION}

This paper is concerned with the existence of solutions and extremal solutions for a perturbed nonlinear boundary value problem with integral boundary conditions. More precisely, we consider the following perturbed nonlinear boundary value problem with integral boundary conditions:

$$
\begin{gathered}
x^{\prime \prime}(t) \in F(t, x(t))+G(t, x(t)) \text { for a.e. } t \in[0,1], \\
x(0)-k_{1} x^{\prime}(0)=\int_{0}^{1} h_{1}(x(s)) d s \\
x(1)+k_{2} x^{\prime}(1)=\int_{0}^{1} h_{2}(x(s)) d s
\end{gathered}
$$

where $F, G:[0,1] \times \mathbb{R} \rightarrow \mathcal{P}(\mathbb{R})$ are compact-valued and multivalued maps, $\mathcal{P}(\mathbb{R})$ is the family of all nonempty subsets of $\mathbb{R}, h_{i}: \mathbb{R} \rightarrow \mathbb{R}$ are continuous functions and $k_{i}$ are nonnegative constants $(i=1,2)$. Boundary value problems with integral boundary conditions form a very interesting and important class of problems. They include two-, three-, multipoint and nonlocal boundary value problems as special cases. For boundary value problems with integral boundary conditions and comments on their importance, we refer the reader to the papers of Brykalov [4], Denche and Marhoune [7], Gallardo [11], Karakostas and Tsamatos [15], Lomtatidze and Malaguti [20], Jankowskii [14], Krall [18] and the references therein. Recently, Khan [16] applied the generalized method of quasilinearization to a class of second order boundary value problems with integral boundary conditions. Belarbi and Benchohra [3] considered the particular problem (1)-(3) with $G \equiv 0$ and obtained existence results when the right-hand 
side has convex as well as nonconvex values. The present paper is motived by a recent one due to Dhage, Gatsori and Ntouyas [10] in which the existence of solutions and extremal solutions for first order perturbed functional differential inclusions is proved under the mixed generalized Lipschitz and Carathéodory conditions. In this paper, we present existence results for the problem (1)-(3) under the mixed generalized Lipschitz and Carathéodory conditions.

\section{Preliminaries}

In this section, we introduce the notation, definitions, and preliminary facts from multivalued analysis which are used throughout this paper.

$C([0,1], \mathbb{R})$ is the Banach space of all continuous functions from $[0,1]$ into $\mathbb{R}$ with the norm

$$
\|x\|_{\infty}=\sup \{|x(t)|: 0 \leq t \leq 1\}
$$

$L^{1}([0,1], \mathbb{R})$ denotes the Banach space of measurable functions $x:[0,1] \longrightarrow \mathbb{R}$ which are Lebesgue integrable normed by

$$
\|x\|_{L^{1}}=\int_{0}^{1}|x(t)| d t \quad \text { for all } x \in L^{1}([0,1], \mathbb{R}) .
$$

$A C^{1}((0,1), \mathbb{R})$ is the space of differentiable functions $x:(0,1) \rightarrow \mathbb{R}$, whose first derivative $x^{\prime}$ is absolutely continuous.

Let $(X,|\cdot|)$ be a normed space, $\mathcal{P}_{c l}(X)=\{Y \in \mathcal{P}(X): Y$ closed $\}, \quad \mathcal{P}_{b}(X)=$ $\{Y \in \mathcal{P}(X): Y$ bounded $\}, P_{c p}(X)=\{Y \in \mathcal{P}(X): Y$ compact $\}$ and $P_{c p, c}(X)=$ $\{Y \in \mathcal{P}(X): Y$ compact and convex $\}$. A multivalued map $G: X \rightarrow \mathcal{P}(X)$ is convex- (closed-) valued if $G(x)$ is convex- (closed-) for all $x \in X$. $G$ is bounded on bounded sets if $G(B)=\cup_{x \in B} G(x)$ is bounded in $X$ for all $B \in \mathcal{P}_{b}(X)$ (i.e., $\left.\sup _{x \in B}\{\sup \{|y|: y \in G(x)\}\}<\infty\right) . G$ is called upper semi-continuous (u.s.c.) on $x \in B$

$X$ if for each $x_{0} \in X$ the set $G\left(x_{0}\right)$ is a nonempty closed subset of $X$ and if for each open set $N$ of $X$ containing $G\left(x_{0}\right)$, there exists an open neighbourhood $N_{0}$ of $x_{0}$ such that $G\left(N_{0}\right) \subseteq N . G$ is said to be completely continuous if $G(\mathcal{B})$ is relatively compact for every $\mathcal{B} \in \mathcal{P}_{b}(X)$. If the multivalued map $G$ is completely continuous with nonempty compact values, then $G$ is u.s.c. if and only if $G$ has a closed graph (i.e., $x_{n} \longrightarrow x_{*}, y_{n} \longrightarrow y_{*}, y_{n} \in G\left(x_{n}\right)$ imply $\left.y_{*} \in G\left(x_{*}\right)\right)$. $G$ has a fixed point if there is $x \in X$ such that $x \in G(x)$. The fixed point set of the multivalued operator $G$ will be denoted by FixG. A multivalued map $G:[0,1] \rightarrow \mathcal{P}_{c l}(\mathbb{R})$ is said to be measurable if for every $y \in \mathbb{R}$, the function $t \longmapsto d(y, G(t))=\inf \{|y-z|: z \in G(t)\}$ is measurable. For more details on multivalued maps see the books of Aubin and Cellina [1], Aubin and Frankowska [2], Deimling [6] and $\mathrm{Hu}$ and Papageorgiou [13] .

Definition 2.1. A multivalued map $F:[0,1] \times \mathbb{R} \rightarrow \mathcal{P}(\mathbb{R})$ is said to be Carathéodory if

(i) $t \longmapsto F(t, y)$ is measurable for each $y \in \mathbb{R}$, and

(ii) $y \longmapsto F(t, y)$ is upper semi-continuous for a.e. $t \in[0,1]$. 
For each $x \in C([0,1], \mathbb{R})$, define the set of selections of $F$ by

$$
S_{F, x}=\left\{v \in L^{1}([0,1], \mathbb{R}): v(t) \in F(t, x(t)) \text { for a.e. } t \in[0,1]\right\} .
$$

Let $F:[0,1] \times \mathbb{R} \rightarrow \mathcal{P}(\mathbb{R})$ be a multivalued map with nonempty compact values. Assign to $F$ the multivalued operator

$$
\mathcal{F}: C([0,1], \mathbb{R}) \rightarrow \mathcal{P}\left(L^{1}([0,1], \mathbb{R})\right)
$$

by letting

$$
\mathcal{F}(x)=\left\{w \in L^{1}([0,1], \mathbb{R}): w(t) \in F(t, x(t)) \text { for a.e. } t \in[0,1]\right\} .
$$

The operator $\mathcal{F}$ is called the Nemytskij operator associated with $F$.

Let $(X, d)$ be a metric space induced from the normed space $(X,|\cdot|)$.

Consider $H_{d}: \mathcal{P}(X) \times \mathcal{P}(X) \longrightarrow \mathbb{R}_{+} \cup\{\infty\}$ given by

$$
H_{d}(A, B)=\max \left\{\sup _{a \in A} d(a, B), \sup _{b \in B} d(A, b)\right\},
$$

where $d(A, b)=\inf _{a \in A} d(a, b), d(a, B)=\inf _{b \in B} d(a, b)$. Then $\left(\mathcal{P}_{b, c l}(X), H_{d}\right)$ is a metric space and $\left(\mathcal{P}_{c l}(X), H_{d}\right)$ is a generalized metric space, see [17].

Definition 2.2. A multivalued operator $N: X \rightarrow \mathcal{P}_{c l}(X)$ is called

a) $\gamma$-Lipschitz if and only if there exists $\gamma>0$ such that

$$
H_{d}(N(x), N(y)) \leq \gamma d(x, y), \quad \text { for each } x, y \in X,
$$

b) a contraction if and only if it is $\gamma$-Lipschitz with $\gamma<1$.

We apply the following form of the fixed point theorem of Dhage [8] in the sequel.

Theorem 2.1. Let $B(0, r)$ and $B[0, r]$ denote respectively the open and the closed ball in a Banach space $E$ centered at the origin and of radius $r$ and let $A: B[0, r] \rightarrow \mathcal{P}_{c l, c v, b d}(E)$ and $B: B[0, r] \rightarrow \mathcal{P}_{c p, c v}(E)$ be two multivalued operators satisfying

(i) $A$ is a multivalued contraction, and

(ii) $B$ is compact and upper semi-continuous.

Then either

(a) the operator inclusion $x \in A x+B x$ has a solution in $B[0, r]$ or

(b) there exists an $u \in E$ with $\|u\|=r$ such that $\lambda u \in A u+B u$ for some $\lambda>1$.

The following lemma will be used in the sequel.

Lemma 2.1 ([19]). Let $X$ be a Banach space. Let $F:[0,1] \times X \longrightarrow \mathcal{P}_{c p, c v}(X)$ be an $L^{1}$-Carathéodory multivalued map with $S_{F, x} \neq \varnothing$ and let $\Gamma$ be a linear continuous mapping from $L^{1}([0,1], X)$ to $C([0,1], X)$, then the operator

$$
\begin{aligned}
\Gamma \circ S_{F}: C([0,1], X) & \longrightarrow \mathcal{P}_{c p, c v}(C([0,1], X)), \\
x & \longmapsto\left(\Gamma \circ S_{F}\right)(x):=\Gamma\left(S_{F, x}\right)
\end{aligned}
$$

is a closed graph operator in $C([0,1], X) \times C([0,1], X)$. 


\section{Existence Result}

In this section, we are concerned with the existence of solutions for the problem (1)-(3).

Definition 3.1. A function $x \in A C^{1}((0,1), \mathbb{R})$ is said to be a solution of (1)(3) if there exist functions $v_{1}, v_{2} \in L^{1}([0,1], \mathbb{R})$ with $v_{1}(t) \in F(t, x(t))$ for a.e. $t \in[0,1]$ and $v_{2}(t) \in G(t, x(t))$ for a.e. $t \in[0,1]$ such that $x^{\prime \prime}(t)=v_{1}(t)+v_{2}(t)$ for a.e. $t \in[0,1]$ and the function $x$ satisfies the conditions (2) and (3).

We need the following auxiliary result. Its proof uses a standard argument.

Lemma 3.1. For any $\sigma(t), \rho_{1}(t), \rho_{2}(t) \in L^{1}([0,1], \mathbb{R})$, the nonhomogeneous linear problem

$$
\begin{gathered}
x^{\prime \prime}(t)=\sigma(t) \text { for a.e. } t \in[0,1], \\
x(0)-k_{1} x^{\prime}(0)=\int_{0}^{1} \rho_{1}(s) d s, \\
x(1)+k_{2} x^{\prime}(1)=\int_{0}^{1} \rho_{2}(s) d s,
\end{gathered}
$$

has a unique solution $x \in A C^{1}((0,1), \mathbb{R})$,

$$
x(t)=P(t)+\int_{0}^{1} H(t, s) \sigma(s) d s
$$

where

$$
P(t)=\frac{1}{1+k_{1}+k_{2}}\left\{\left(1-t+k_{2}\right) \int_{0}^{1} \rho_{1}(s) d s+\left(k_{1}+t\right) \int_{0}^{1} \rho_{2}(s) d s\right\}
$$

is the unique solution of the problem

$$
\begin{aligned}
& x^{\prime \prime}(t)=0 \text { for a.e. } t \in[0,1], \\
& x(0)-k_{1} x^{\prime}(0)=\int_{0}^{1} \rho_{1}(s) d s, \\
& x(1)+k_{2} x^{\prime}(1)=\int_{0}^{1} \rho_{2}(s) d s,
\end{aligned}
$$

and

$$
H(t, s)=\frac{-1}{k_{1}+k_{2}+1} \begin{cases}\left(k_{1}+t\right)\left(1-s+k_{2}\right), & 0 \leq t<s \leq 1, \\ \left(k_{1}+s\right)\left(1-t+k_{2}\right), & 0 \leq s<t \leq 1,\end{cases}
$$

is the Green's function of the corresponding homogeneous problem. We note that $H(t, s)<0$ on $(0,1) \times(0,1)$. 
We transform the BVP (1)-(3) into a fixed point problem. Consider the operator $N: C([0,1], \mathbb{R}) \longrightarrow \mathcal{P}(C([0,1], \mathbb{R}))$ defined by

$$
\begin{gathered}
N(x)=\left\{u \in C([0,1], \mathbb{R}): u(t)=\operatorname{Px}(t)+\int_{0}^{1} H(t, s)\left(v_{1}(s)+v_{2}(s)\right) d s,\right. \\
\left.v_{1} \in S_{F, x} \text { and } v_{2} \in S_{G, x}\right\} .
\end{gathered}
$$

where the operator $P: A C^{1}(J, \mathbb{R}) \rightarrow \mathbb{R}$ is defined by

$$
P x(t)=\frac{1}{1+k_{1}+k_{2}}\left\{\left(1-t+k_{2}\right) \int_{0}^{1} h_{1}(x(s)) d s+\left(k_{1}+t\right) \int_{0}^{1} h_{2}(x(s)) d s\right\} .
$$

Remark 3.1. Clearly, from Lemma 3.1, the fixed points of $N$ are solutions of (1) $-(3)$.

Let us introduce the following hypotheses:

(H1) The function $t \rightarrow F(t, y)$ is measurable, convex-valued and integrably bounded for each $y \in \mathbb{R}$.

(H2) $H_{d}(F(t, y), F(t, \bar{y})) \leq l(t)|y-\bar{y}|$ for a.e. $t \in[0,1]$ and all $y, \bar{y} \in \mathbb{R}$ where $l \in L^{1}([0,1], \mathbb{R})$ and $H_{d}(0, F(t, 0)) \leq l(t)$ for a.e. $t \in[0,1]$.

(H3) There exist two nonnegative constants $d_{1}$ and $d_{2}$ such that

$$
\begin{gathered}
\left|h_{1}(y)-h_{1}(\bar{y})\right| \leq d_{1}|y-\bar{y}| \text { and }\left|h_{2}(y)-h_{2}(\bar{y})\right| \leq d_{2}|y-\bar{y}| \\
\text { for all } y, \bar{y} \in \mathbb{R} .
\end{gathered}
$$

(H4) The multivalued map $G(t, y)$ has compact and convex values for each $(t, y) \in[0,1] \times \mathbb{R}$.

(H5) $G$ is Carathéodory.

(H6) There exist a function $q \in L^{1}([0,1], \mathbb{R})$ with $q(t)>0$ for a.e. $t \in[0,1]$ and a continuous nondecreasing function $\psi: \mathbb{R}_{+} \rightarrow(0, \infty)$ such that

$$
\|G(t, y)\|_{\mathcal{P}} \leq q(t) \psi(|y|) \text { for a.e. } t \in[0,1] \text { for all } y \in \mathbb{R}
$$

(H7) There exists a real number $r>0$ such that

$$
\begin{aligned}
& r>\frac{\frac{1}{1+k_{1}+k_{2}}\left[\left(1+k_{2}\right) h_{1}(r)+\left(1+k_{1}\right) h_{2}(r)\right]+H^{*}\|l\|_{L^{1}}+H^{*} \psi(r)\|q\|_{L^{1}}}{1-H^{*}\|l\|_{L^{1}}}, \\
& \quad \text { where } H *=\sup _{(t, s) \in[0,1] \times[0,1]}|H(t, s)| .
\end{aligned}
$$

Theorem 3.1. Suppose that hypotheses $(\mathrm{H} 1)-(\mathrm{H} 7)$ are satisfied. If

$$
\frac{1}{1+k_{1}+k_{2}}\left[\left(1+k_{1}\right) d_{1}+\left(1+k_{2}\right) d_{2}\right]+H^{*}\|l\|_{L^{1}}<1
$$

then the BVP (1)-(3) has at least one solution. 
Proof. Let $X=C([0,1], \mathbb{R})$ and define an open ball $B(0, r)$ in $X$ centered at the origin and of radius $r$, where the real number $r$ satisfies the inequality in hypothesis (H7). Define two multivalued maps on $B[0, r]$ by

$$
A(x)=\left\{u \in X: u(t)=P x(t)+\int_{0}^{1} H(t, s) v_{1}(s) d s, \quad v_{1} \in S_{F, x}\right\}
$$

and

$$
B(x)=\left\{u \in X: u(t)=\int_{0}^{1} H(t, s) v_{2}(s) d s, \quad v_{2} \in S_{G, x}\right\} .
$$

We shall show that the operators $A$ and $B$ satisfy all the conditions of Theorem 2.1. The proof will be given in several steps.

Step 1: First we show that $A(x)$ is a closed convex and bounded subset of $X$ for each $x \in B[0, r]$. This follows easily if we show that the values of the Nemytskij operator associated are closed in $L^{1}([0,1], \mathbb{R})$. Let $\left\{w_{n}\right\}$ be a sequence in $L^{1}([0,1], \mathbb{R})$ converging to a point $w$. Then $w_{n} \rightarrow w$ in measure and thus there exists a subset $S$ of positive integers with $\left\{w_{n}\right\}$ converging a.e. to $w$ as $n \rightarrow \infty$ through $S$. Now, since (H1) holds, the values of $S_{F, x}$ are closed in $L^{1}([0,1], \mathbb{R})$. Thus, for each $x \in B[0, r]$, we have that $A(x)$ is a nonempty and closed subset of $X$.

We prove that $A(x)$ is a convex subset of $X$ for each $x \in B[0, r]$. Let $u_{1}, u_{2} \in$ $A(x)$. Then there exist $v_{1}, v_{2} \in S_{F, x}$ such that for each $t \in[0,1]$ we have

$$
u_{i}(t)=P x(t)+\int_{0}^{1} H(t, s) v_{i}(s) d s \quad(i=1,2) .
$$

Let $0 \leq d \leq 1$. Then, for each $t \in[0,1]$, we obtain

$$
\left(d u_{1}+(1-d) u_{2}\right)(t)=\operatorname{Px}(t)+\int_{0}^{1} H(t, s)\left[d v_{1}(s)+(1-d) v_{2}(s)\right] d s .
$$

Since $S_{F, x}$ is convex (because $F$ has convex values), we have

$$
d u_{1}+(1-d) u_{2} \in A(x) .
$$

Step 2: We show that $A$ is a multivalued contraction on $B[0, r]$. Let $x, \bar{x} \in$ $B[0, r]$ and $u_{1} \in A(x)$. Then there exists $v_{1}(t) \in F(t, x(t))$ such that for each $t \in[0,1]$

$$
u_{1}(t)=P x(t)+\int_{0}^{1} H(t, s) v_{1}(s) d s .
$$

From (H2) it follows that

$$
H_{d}(F(t, x(t)), F(t, \bar{x}(t))) \leq l(t)|x(t)-\bar{x}(t)| .
$$


Hence there exists $w \in F(t, \bar{x}(t))$ such that

$$
\left|v_{1}(t)-w\right| \leq l(t)|x(t)-\bar{x}(t)|, \quad t \in[0,1]
$$

Consider $U:[0,1] \rightarrow \mathcal{P}(\mathbb{R})$ given by

$$
U(t)=\left\{w \in \mathbb{R}:\left|v_{1}(t)-w\right| \leq l(t)|x(t)-\bar{x}(t)|\right\} .
$$

Since the multivalued operator $V(t)=U(t) \cap F(t, \bar{x}(t))$ is measurable (see Proposition III.4 in [5]), there exists a function $v_{2}(t)$ which is a measurable selection for $V$. So, $v_{2}(t) \in F(t, \bar{x}(t))$ and for each $t \in[0,1]$

$$
\left|v_{1}(t)-v_{2}(t)\right| \leq l(t)|x(t)-\bar{x}(t)| .
$$

Let us define for each $t \in[0,1]$

$$
u_{2}(t)=P \bar{x}(t)+\int_{0}^{1} H(t, s) v_{2}(s) d s
$$

where

$$
P \bar{x}(t)=\frac{1}{1+k_{1}+k_{2}}\left[\left(1-t+k_{2}\right) \int_{0}^{1} h_{1}(\bar{x}(s)) d s+\left(1+k_{1}\right) \int_{0}^{1} h_{2}(\bar{x}(s)) d s\right] .
$$

We have

$$
\begin{aligned}
\left|u_{1}(t)-u_{2}(t)\right| \leq & |P x(t)-P \bar{x}(t)|+\int_{0}^{1}|H(t, s)|\left|v_{1}(s)-v_{2}(s)\right| d s \\
\leq & \frac{1}{1+k_{1}+k_{2}}\left[\left(1+k_{1}\right) d_{1}+\left(1+k_{2}\right) d_{2}\right]\|x-\bar{x}\|_{\infty} \\
& +\int_{0}^{1}|H(t, s)| l(s)|x(s)-\bar{x}(s)| d s .
\end{aligned}
$$

Thus

$$
\left\|u_{1}-u_{2}\right\| \leq\left(\frac{1}{1+k_{1}+k_{2}}\left[\left(1+k_{1}\right) d_{1}+\left(1+k_{2}\right) d_{2}\right]+H^{*}\|l\|_{L^{1}}\right)\|x-\bar{x}\|_{\infty} .
$$

From an analogous relation obtained by interchanging $x$ and $\bar{x}$, it follows that $H_{d}(A(x), A(\bar{x})) \leq\left(\frac{1}{1+k_{1}+k_{2}}\left[\left(1+k_{1}\right) d_{1}+\left(1+k_{2}\right) d_{2}\right]+H^{*}\|l\|_{L^{1}}\right)\|x-\bar{x}\|_{\infty}$.

So, $A$ is a multivalued contraction on $X$.

Step 3: Now we show that the multivalued operator $B$ is compact and upper semi-continuous on $B[0, r]$. First we show that $B$ is compact on $B[0, r]$. Let 
$x \in B[0, r]$ be arbitrary. Then for each $u \in B(x)$ there exists $v \in S_{G, x}$ such that for each $t \in[0,1]$ we have

$$
u(t)=\int_{0}^{1} H(t, s) v(s) d s .
$$

From (H5) we have

$$
\begin{aligned}
|u(t)| & \leq \int_{0}^{1}|H(t, s)||v(s)| d s \leq H^{*} \int_{0}^{1}|v(s)| d s \\
& \leq H^{*} \int_{0}^{1} q(s) \psi\left(\|x\|_{\infty}\right) d s \leq H^{*}\|q\|_{L^{1}} \psi(r) .
\end{aligned}
$$

Next, we show that $B$ maps the bounded sets into the equi-continuous sets of $X$. Let $t, \tau \in[0,1]$, and $x \in B[0, r]$. For each $u \in B(x)$

$$
\begin{aligned}
|u(t)-u(\tau)| & \leq \int_{0}^{1}|H(t, s)-H(\tau)||v(s)| d s \\
& \leq \int_{0}^{1}|H(t, s)-H(\tau)| q(t) \psi\left(\|x\|_{\infty}\right) d s \\
& \leq \int_{0}^{1}|H(t, s)-H(\tau, s)| q(s) \psi(r) d s .
\end{aligned}
$$

The right-hand side tends to zero as $|t-\tau| \rightarrow 0$. An application of the ArzeláAscoli theorem implies that the operator $B: B[0, r] \longrightarrow \mathcal{P}(X)$ is compact.

Step 4: Next we prove that $B$ has a closed graph. Let $x_{n} \rightarrow x_{*}, y_{n} \in B\left(x_{n}\right)$ and $y_{n} \rightarrow y_{*}$. We need to show that $y_{*} \in B\left(x_{*}\right)$.

$y_{n} \in B\left(x_{n}\right)$ means that there exists $v_{n} \in S_{G, x_{n}}$ such that for each $t \in[0,1]$

$$
y_{n}(t)=\int_{0}^{1} H(t, s) v_{n}(s) d s .
$$

We must show that there exists $y_{*} \in S_{G, x_{*}}$ such that for each $t \in[0,1]$

$$
y_{*}(t)=\int_{0}^{1} H(t, s) v_{*}(s) d s .
$$

Clearly, we have

$$
\left\|y_{n}-y_{*}\right\|_{\infty} \longrightarrow 0 \quad \text { as } \quad n \rightarrow \infty
$$

Consider the continuous linear operator

$$
\Gamma: L^{1}([0,1], \mathbb{R}) \rightarrow X
$$


given by

$$
v \longmapsto(\Gamma v)(t)=\int_{0}^{1} H(t, s) v(s) d s .
$$

From Lemma 2.1 it follows that $\Gamma \circ S_{F}$ is a closed graph operator. Moreover, we have

$$
y_{n}(t) \in \Gamma\left(S_{G, x_{n}}\right) .
$$

Since $x_{n} \rightarrow x_{*}$, it follows from Lemma 2.1 that

$$
y_{*}(t)=\int_{0}^{1} H(t, s) v_{*}(s) d s
$$

for some $v_{*} \in S_{G, x_{*}}$.

Step 5 : Now we show that the second assertion of Theorem 2.1 is not true. Let $u \in X$ be a possible solution of $\lambda u \in A(u)+B(u)$ for some real number $\lambda>1$ with $\|u\|_{\infty}=r$. Then there exist $v_{1} \in S_{F, u}$ and $v_{2} \in S_{G, u}$ such that for each $t \in[0,1]$ we have

$$
u(t)=\lambda^{-1} P x(t)+\lambda^{-1} \int_{0}^{1} H(t, s) v_{1}(s) d s+\lambda^{-1} \int_{0}^{1} H(t, s) v_{2}(s) d s .
$$

Then

$$
\begin{aligned}
|u(t)| \leq & |P x(t)|+H^{*} \int_{0}^{1}\left|v_{1}(s)\right| d s+H^{*} \int_{0}^{1}\left|v_{2}(s)\right| d s \\
\leq & \frac{1}{1+k_{1}+k_{2}}\left[\left(1+k_{2}\right) \int_{0}^{1} h_{1}(u(s)) d s+\left(1+k_{1}\right) \int_{0}^{1} h_{2}(u(s)) d s\right] \\
& +H^{*}(l(s)|u(s)|+l(s)) d s+H^{*} \int_{0}^{1} q(s) \psi(|u(s)|) d s \\
\leq & \frac{1}{1+k_{1}+k_{2}}\left[\left(1+k_{2}\right) \int_{0}^{1} h_{1}\left(\|u\|_{\infty}\right) d s+\left(1+k_{1}\right) \int_{0}^{1} h_{2}\left(\|u\|_{\infty}\right) d s\right] \\
& +H^{*}\left(l(s)\|u\|_{\infty}+l(s)\right) d s+H^{*} \int_{0}^{1} q(s) \psi\left(\|u\|_{\infty}\right) d s .
\end{aligned}
$$


Taking the supremum over $t$ we get

$$
\begin{aligned}
\|u\|_{\infty} \leq & \frac{1}{1+k_{1}+k_{2}}\left[\left(1+k_{2}\right) \int_{0}^{1} h_{1}\left(\|u\|_{\infty}\right) d s+\left(1+k_{1}\right) \int_{0}^{1} h_{2}\left(\|u\|_{\infty}\right) d s\right] \\
& +H^{*} \int_{0}^{1}\left(l(s)\|u\|_{\infty}+l(s)\right) d s+H^{*} \int_{0}^{1} q(s) \psi\left(\|u\|_{\infty}\right) d s .
\end{aligned}
$$

Substituting $\|u\|_{\infty}=r$ into the above inequality yields

$$
r \leq \frac{\frac{1}{1+k_{1}+k_{2}}\left[\left(1+k_{2}\right) h_{1}(r)+\left(1+k_{1}\right) h_{2}(r)\right]+H^{*}\|l\|_{L^{1}}+H^{*} \psi(r)\|q\|_{L^{1}}}{1-H^{*}\|l\|_{L^{1}}}
$$

which is a contradiction to (H7). As a result, the conclusion (ii) of Theorem 2.1 does not hold. Hence the conclusion (i) holds and consequently the BVP (1)-(3) has a solution $x$ on $[0,1]$. This completes the proof.

\section{Existence of Extremal Solutions}

In this section we shall prove the existence of maximal and minimal solutions of the BVP (1)-(3) under suitable monotonicity conditions on the multifunctions involved in it.

We equip the space $X=C([0,1], \mathbb{R})$ with the order relation $\leq$ defined by the cone $K$ in $X$, that is,

$$
K=\{x \in X \mid x(t) \geq 0, \quad \forall t \in[0,1]\} .
$$

It is known that the cone $K$ is normal in $X$. The details on cones and their properties can be found in Heikkila and Lakshmikantham [12]. Let $a, b \in X$ be such that $a \leq b$. Then, by an order interval $[a, b]$ we mean a set of points in $X$ given by

$$
[a, b]=\{x \in X \mid a \leq x \leq b\} .
$$

Let $D, Q \in \mathcal{P}_{c l}(X)$. Then by $D \leq Q$ we mean $a \leq b$ for all $a \in D$ and $b \in Q$. Thus $a \leq D$ implies that $a \leq b$ for all $b \in Q$; in particular, if $D \leq D$, then it follows that $D$ is a singleton set.

Definition 4.1. Let $X$ be an ordered Banach space. A mapping $T: X \rightarrow$ $\mathcal{P}_{c l}(X)$ is called isotone increasing if $x, y \in X$ with $x<y$, then we have that $T(x) \leq T(y)$. Similarly, $T$ is called isotone decreasing if $T(x) \geq T(y)$ whenever $x<y$.

We use the following fixed point theorem in the proof of the main existence result of this section.

Theorem 4.1 (Dhage [9]). Let $[a, b]$ be an order interval in a Banach space and let $A, B:[a, b] \rightarrow \mathcal{P}_{c l}(X)$ be two multivalued operators satisfying the conditions:

(a) A is a multivalued contraction,

(b) $B$ is completely continuous, 
(c) $A$ and $B$ are strictly monotone increasing, and

(d) $A(x)+B(x) \subset[a, b], \forall x \in[a, b]$.

Further if the cone $K$ in $X$ is normal, then the operator inclusion $x \in A(x)+$ $B(x)$ has the smallest fixed point $x_{*}$ and the largest fixed point $x^{*} \in[a, b]$. Moreover $x_{*}=\lim _{n} x_{n}$ and $x^{*}=\lim _{n} y_{n}$, where $\left\{x_{n}\right\}$ and $\left\{y_{n}\right\}$ are the sequences in $[a, b]$ defined by

$$
x_{n+1} \in A\left(x_{n}\right)+B\left(x_{n}\right), x_{0}=a \text { and } y_{n+1} \in A\left(y_{n}\right)+B\left(y_{n}\right), y_{0}=b .
$$

We need the following definitions in the sequel.

Definition 4.2. A function $a \in A C^{1}([0,1], \mathbb{R})$ is called a strict lower solution of the $\operatorname{BVP}(1)-(3)$ if $a^{\prime \prime}(t) \geq v_{1}(t)+v_{2}(t)$ for a.e. $t \in[0,1]$,

$$
a(0)-k_{1} a^{\prime}(0) \leq \int_{0}^{1} h_{1}(a(s)) d s \text { and } a(1)+k_{2} a^{\prime}(1) \leq \int_{0}^{1} h_{2}(a(s)) d s,
$$

where $v_{1}, v_{2} \in L^{1}([0,1], \mathbb{R})$ are such that $v_{1}(t) \in F(t, a(t))$ and $v_{2}(t) \in G(t, a(t))$ for a.e. $t \in[0,1]$.

Similarly, a strict upper solution $b$ of BVP (1)-(3) is defined by reversing the order.

Definition 4.3. A solution $x_{M}$ of the BVP (1)-(3) is said to be maximal if for any other solution $x$ of $\operatorname{BVP}(1)-(3)$ on $[0,1]$ we have $x(t) \leq x_{M}(t)$ for each $t \in[0,1]$.

Similarly, a minimal solution of the BVP (1)-(3) is defined by reversing the order.

Definition 4.4. A multivalued function $F(t, x)$ is called strictly monotone increasing in $x$ almost everywhere for $t \in J$, if $F(t, x) \leq F(t, y)$ for a.e. $t \in J$ for all $x, y \in \mathbb{R}$ with $x<y$. Similarly $F(t, x)$ is called strictly monotone decreasing in $x$ almost everywhere for $t \in J$, if $F(t, x) \geq F(t, y)$ for a.e. $t \in J$ for all $x, y \in \mathbb{R}$ with $x<y$.

We consider the following assumptions in the sequel.

(H8) The multivalued functions $F(t, x)$ and $G(t, x)$ are strictly monotone decreasing in $x$ for a.e. $t \in[0,1]$.

(H9) The functions $h_{1}$ and $h_{2}$ are continuous and nondecreasing.

(H10) The BVP (1)-(3) has a lower solution $a$ and an upper solution $b$ with $a \leq b$.

Theorem 4.2. Assume that the hypotheses (H1)-(H5), (H8)-(H10) hold. Then the BVP (1)-(3) has a minimal and a maximal solution on $[0,1]$.

Proof. It can be shown, as in the proof of Theorem 3.1, that $A$ and $B$ define the multivalued operators $A:[a, b] \rightarrow \mathcal{P}_{c l, c v, b d}(X)$ and $B:[a, b] \rightarrow \mathcal{P}_{c p, c v}(X)$. It can be similarly shown that $A$ and $B$ are respectively a multivalued contraction and compact and upper semi-continuous on $[a, b]$. We shall show that $A$ and $B$ 
are isotone increasing on $[a, b]$. Let $x, y \in[a, b]$ be such that $x \leq y, x \neq y$. Then by (H8) and (H9) we have for each $t \in[0,1]$

$$
\begin{aligned}
A(x)= & \left\{u \in X: u(t)=P x(t)+\int_{0}^{1} H(t, s) v_{1}(s) d s, v_{1} \in S_{F, x}\right\} \\
= & \left\{u \in X: u(t)=\frac{1}{1+k_{1}+k_{2}}\left[\left(1-t+k_{2}\right) \int_{0}^{1} h_{1}(x(s)) d s\right.\right. \\
& \left.\left.+\left(t+k_{1}\right) \int_{0}^{1} h_{2}(x(s)) d s\right]+\int_{0}^{1} H(t, s) v_{1}(s) d s, v_{1} \in S_{F, x}\right\} \\
\leq & \left\{u \in X: u(t)=\frac{1}{1+k_{1}+k_{2}}\left[\left(1-t+k_{2}\right) \int_{0}^{1} h_{1}(y(s)) d s\right.\right. \\
& \left.\left.+\left(t+k_{1}\right) \int_{0}^{1} h_{2}(y(s)) d s\right]+\int_{0}^{1} H(t, s) v_{1}(s) d s, v_{1} \in S_{F, y}\right\} \\
= & A(y) .
\end{aligned}
$$

Hence $A(x) \leq A(y)$.

Similarly, by (H8) we have for each $t \in[0,1]$

$$
\begin{aligned}
B(x) & =\left\{u \in X: u(t)=\int_{0}^{1} H(t, s) v_{2}(s) d s, \quad v_{2} \in S_{G, x}\right\} \\
& \leq\left\{u \in X: u(t)=\int_{0}^{1} H(t, s) v_{2}(s) d s, \quad v_{2} \in S_{G, y}\right\} \\
& =B(y) .
\end{aligned}
$$

Hence $B(x) \leq B(y)$. Thus $A$ and $B$ are isotone increasing on $[a, b]$. Finally, let $x \in[a, b]$ be any element. Then by (H10),

$$
a \leq A(a)+B(a) \leq A(x)+B(x) \leq A(b)+B(b) \leq b,
$$

which shows that $A(x)+B(x) \subset[a, b]$ for all $x \in[a, b]$. Thus the multivalued operators $A$ and $B$ satisfy all the conditions of Theorem 4.1 to yield that the BVP (1)-(3) has a maximal and a minimal solution on $[0,1]$. This completes the proof.

\section{REFERENCES}

1. J.-P. Aubin and A. Cellina, Differential inclusions. Set-valued maps and viability theory. Grundlehren der Mathematischen Wissenschaften [Fundamental Principles of Mathematical Sciences], 264. Springer-Verlag, Berlin, 1984. 
2. J.-P. Aubin and H. Frankowska, Set-valued analysis. Systems \& Control: Foundations \& Applications, 2. Birkhäuser Boston, Inc., Boston, MA, 1990.

3. A. Belarbi and M. Benchohra, Existence results for nonlinear boundary-value problems with integral boundary conditions. Electron. J. Differential Equations 2005, No. 06, 10 pp. (electronic).

4. S. A. BRykAlov, A Second-order nonlinear problem with two-point and integral boundary conditions. Georgian Math. J. 1(1994), No. 3, 243-249.

5. C. Castaing and M. Valadier, Convex analysis and measurable multifunctions. Lecture Notes in Mathematics, Vol. 580. Springer-Verlag, Berlin-New York, 1977.

6. K. Deimling, Multivalued differential equations. de Gruyter Series in Nonlinear Analysis and Applications, 1. Walter de Gruyter \& Co., Berlin, 1992.

7. M. Denche and A. L. Marhoune, High-order mixed-type differential equations with weighted integral boundary conditions. Electron. J. Differential Equations 2000, No. 60, 10 pp. (electronic).

8. B. C. Dhage, Multivalued mappings and fixed points. II. Tamkang J. Math. 36(2006) (to appear).

9. B. C. Dhage, A fixed point theorem for multi-valued mappings in ordered Banach spaces with applications. II. Panamer. Math. J. 15(2005), No. 3, 15-34.

10. B. C. Dhage, E. Gatsori, and S. K. Ntouyas, Existence theory for perturbed functional differential inclusions. Med. J. Math. (to appear).

11. J. M. Gallardo, Second-order differential operators with integral boundary conditions and generation of analytic semigroups. Rocky Mountain J. Math. 30(2000), No. 4, 12651291.

12. S. Heikkilä and V. Lakshmikantham, V. Monotone iterative techniques for discontinuous nonlinear differential equations. Monographs and Textbooks in Pure and Applied Mathematics, 181. Marcel Dekker, Inc., New York, 1994.

13. Sh. Hu and N. S. Papageorgiou, Handbook of multivalued analysis. Vol. I. Theory. Mathematics and its Applications, 419. Kluwer Academic Publishers, Dordrecht, 1997.

14. T. Jankowskin, Differential equations with integral boundary conditions. J. Comput. Appl. Math. 147(2002), No. 1, 1-8.

15. G. L. Karakostas and P. Ch. Tsamatos, Multiple positive solutions of some Fredholm integral equations arisen from nonlocal boundary-value problems. Electron. J. Differential Equations 2002, No. 30, 17 pp. (electronic).

16. R. A. KHAN, The generalized method of quasilinearization and nonlinear boundary value problems with integral boundary conditions. Electron. J. Qual. Theory Differ. Equ. 2003, No. 19, 15 pp. (electronic).

17. M. KisielewiCz, Differential inclusions and optimal control. Mathematics and its Applications (East European Series), 44. Kluwer Academic Publishers Group, Dordrecht; PWN-Polish Scientific Publishers, Warsaw, 1991.

18. A. M. KRALL, The adjoint of a differential operator with integral boundary conditions. Proc. Amer. Math. Soc. 16(1965), 738-742.

19. A. Lasota and Z. Opial, An application of the Kakutani-Ky Fan theorem in the theory of ordinary differential equations. Bull. Acad. Polon. Sci. Sr. Sci. Math. Astronom. Phys. 13(1965), 781-786.

20. A. Lomtatidze and L. Malaguti, On a nonlocal boundary value problem for second order nonlinear singular differential equations. Georgian Math. J. 7(2000), No. 1, 133-154. 
(Received 8.03.2005)

Authors' addresses:

A. Belarbi and M. Benchohra

Laboratoire de Mathématiques

Université de Sidi Bel Abbès

BP 89, 22000, Sidi Bel Abbès, Algérie

E-mail: aek_belarbi@yahoo.fr

benchohra@yahoo.com

B. C. Dhage

Kasubai, Gurukul Colony, Ahmedpur-413 515

Dist: Latur Maharashtra, India

E-mail: bcd20012001@yahoo.co.in 